\title{
An Experimental Analysis of Seven Level Cascaded H-Bridge Multilevel inverter for Harmonic Minimization
}

\author{
K.Mahendran ${ }^{1}$, Dr.S.U.Prabha ${ }^{2}$ \\ Assistant Professor, Dept. of Electrical and Electronics Engineering, Jansons Institute of technology, Coimbatore. ${ }^{1}$ \\ Professor, Department of Electrical and Electronics Engineering, Sri Ramakrishna Engineering College, Coimbatore. ${ }^{2}$
}

\begin{abstract}
This research on cascade H-Bridge multilevel inverter in these days for various sectors goes on rapidly due to its systematic operation. This paper proposes a harmonic minimization in distribution network by using power converter. This project deals simulation and experimentation of 7-level inverter. It presents 7-level inverter with harmonics reduction along with the reduction in number of switches. The percentage (\%) total harmonic distortion is calculated for 7-level inverter. A standard cascade multilevel inverter requires $\mathrm{n}$ DC sources for $2 \mathrm{n}+1$ level. The functionality verification of the 7-level inverter is done using MATLAB and HARDWARE. The result of simulation is verified by experimentation. The harmonic components of the output voltage and switching losses can be diminished considerably.
\end{abstract}

Key words: Harmonic, inverter, voltage, Dc Source.

\section{INTRODUCTION}

Tremendous industrial applications have initiated using high power conversion tenders in recent decades. Nearly low and medium voltage motor drives and efficacy applications require low, medium voltage and high power level for a medium voltage grid; it is worrying to connect directly a single switch. As a result, multilevel power converter (both rectifiers and inverters), has been introduced as an alternative in high power and medium voltage situations.

The model of multilevel inverters has been introduced later 1975. The term multilevel starts with the three level inverters. Multilevel inverters include an array of power semiconductors and capacitor voltage sources, the output of which generates voltages with stepped waveforms with less distortion, less switching frequency, higher efficiency, lower voltage devices and better electro-magnetic compatibility. A multilevel inverter not only archives high power ratings, but also enables the use of renewable energy sources. Renewable energy sources such as photo voltaic, wind and fuel cells can be easily interfaced to a multilevel inverter (converter) system for a high power application. The multilevel power converters can be referred as voltage synthesizers in which high output voltage is synthesized from many discrete smaller voltages levels.

The advantage of multilevel converters when compared to other converters can be listed as follows: they can generate output voltages with extremely low distortion and lower $(\mathrm{dv} / \mathrm{dt})$, they draw input current with very low distortion, they can operate with a lower switching frequency, their efficiency is high (>98\%) because of the minimum switching frequency, they are suitable for medium to high power applications, multilevel waveform naturally limits the problem of large voltage transients. The selection of the best multilevel topology for each application is often not clear and is subject to various engineering tradeoffs. The different multilevel topologies are, Diode-clamped multilevel inverter, Capacitor-clamped multilevel inverter and Cascaded multilevel inverter.

\section{KINDS OF MULTILEVEL INVERTER}

\section{Diode Clamped Multilevel Inverter}

The first practical multilevel topology is the neutral point clamped PWM technology first introduced by Nabe.etal in 1980. For m-level inverter, dc bus voltage is splits into ' $\mathrm{m}$ ' levels by $(\mathrm{m}-1)$ series connected bulk capacitors. Here, diodes clamp the switch voltage to half the level of the dc bus voltage, which is an added advantage of this type. This multilevel inverter has some disadvantages than the cascade multilevel inverter. But it has some advantages as follows. This multilevel inverter is difficult compare than cascade multilevel inverter. Excessive clamping diodes are required when levels are high. The issue of maintaining the charge balance of the capacitors is still an open issue for NPC topologies with more than three levels.

\section{Flying Capacitor Multilevel Inverter}

This topology was first proposed in 1992 and is considered to be the serious alternative to the diode clamped topology. In addition to improving the waveform quality, these multilevel inverters substantially reduce voltage stress on the devices. However in this type of inverters the required voltage blocking capabilities of the clamping diodes at higher levels is high. So an alternative multilevel structure where the voltage across an open switch is constrained by clamping diodes has been proposed by Meynard. These inverters are commonly known as flying capacitor. This makes the topology attractive even for the $\mathrm{dc} / \mathrm{dc}$ conversion. At the present time it seems that this topology has few advantages like: Large amount of storage capacitors can provide capabilities during power 
outages, like diode clamped inverters, when the number of $\bullet \mathrm{O} / \mathrm{p}$ voltage Waveforms

levels is high enough, the harmonic content is low enough to avoid the need for filters, and both real and reactive power flow can be controlled. Some of the disadvantages of flying capacitor topology are: Excessive number of storage capacitors is required, the inverter control can be very complicated.

\section{Cascaded Multilevel Inverter}

This topology employs a cascade of low voltage H-bridges each with independent and isolated dc sources. The phase output voltage is synthesized by the sum of series of $\mathrm{H}$ bridges plus one. This cascade multi-level inverter has more capacity compare than other multilevel inverter. One major advantage of this approach is that the number of the output can be further increased without addition of any new components, requiring only the dc sources with different voltage levels. The number of possible output voltage levels is more than twice the number of dc sources $(\mathrm{m}=2 \mathrm{~s}+1)$

The series of H-bridges makes for modularized layout and packaging. This will enable the manufacturing process to be done more quickly and cheaply. Separate dc sources are required for each of the $\mathrm{H}$-bridges. This will limit its application to products that already have multiple SDCSs readily available.

\section{HARMONIC MINATION IN CMLI}

Multilevel inverters are source of high power, often used in industrial applications and can use either sine or modified sine waves. Instead of using one converter to convert an AC current into a DC current, a multilevel inverter uses a series of semiconductor power converters (usually two to three) thus generating higher voltage. While an inverter would transfer energy with the flip of one switch, with a help of multilevel inverter we have to flip several switches, each switch requiring a driving circuit. These multiple switches and circuits usually make multilevel inverters more expensive than normal inverters.

By new approach the disadvantages of the existing methods are minimized .They are,

\section{Harmonic Distortion.}

Switching losses.

Improve the Electro Magnetic compatibility.

Higher voltage capability.

\section{A. Modified Harmonic Distortion}

By this new approach the Harmonics are reduced by 4.93 to 4.7 The harmonics is nothing but the disturbance in the waveforms it may generate from the non-sinusoidal currents in the diode rectifier.

\section{Technique used}

The PWM technique method of single stage H-bridge inverter converts from $\mathrm{DC}$ to $\mathrm{AC}$. The fixed $\mathrm{DC}$ input voltage is converted into a variable $\mathrm{AC}$ output voltage which can be obtained by varying the control of inverter. The AT mega8L-8pu controller provides PWM pulses to MOSFET switches. By using this techniques it improves the,
- Low THD

- Reduces the conversional losses

\section{Pulse-width modulation}

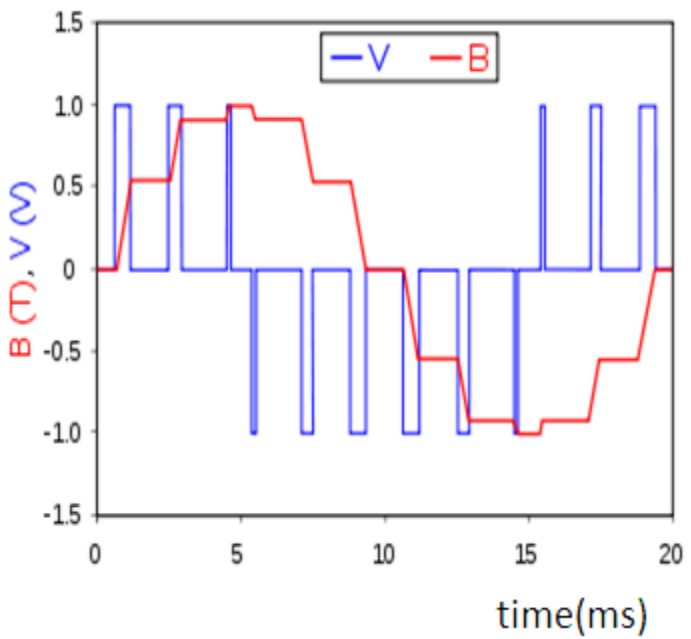

Figure 1 pulse width modulation

Pulse-width modulation (PWM), or pulse-duration modulation (PDM), is a commonly used technique for controlling power to inertial electrical devices, made practical by modern electronic power switches. The average value of voltage (and current) fed to the load is controlled by turning the switch between supply and load on and off at a fast pace. The longer the switch is on compared to the off periods, the higher the power supplied to the load.

The PWM switching frequency has to be much faster than what would affect the load, which is to say the device that uses the power. Typically switching have to be done several times a minute in an electric stove, $120 \mathrm{~Hz}$ in a lamp dimmer, from few kilohertz $(\mathrm{kHz})$ to tens of $\mathrm{kHz}$ for a motor drive and well into the tens or hundreds of $\mathrm{kHz}$ in audio amplifiers and computer power supplies. The term duty cycle describes the proportion of 'on' time to the regular interval or 'period' of time; a low duty cycle corresponds to low power, because the power is off for most of the time. Duty cycle is expressed in percent, $100 \%$ being fully on. The main advantage of PWM is that power loss in the switching devices is very low. When a switch is off there is practically no current, and when it is on, there is almost no voltage drop across the switch. Power loss, being the product of voltage and current, is thus in both cases close to zero. PWM also works well with digital controls, which, because of their on/off nature, can easily set the needed duty cycle.

Block Diagram of the System:

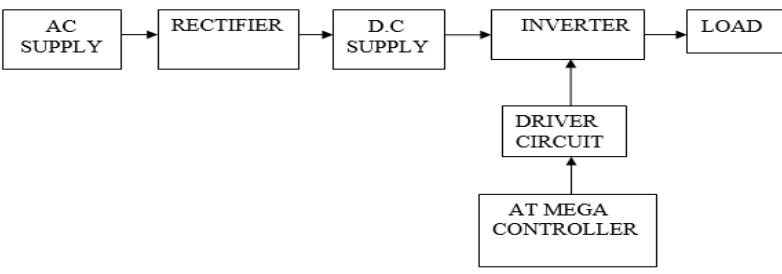

Figure 2. Block diagram of a multilevel level inverter 
The block diagram shows the proposed method of the cascade H-bridge multilevel inverter. In which the AC power is converted into the DC by using the Bridge rectifier. The DC source is used in inverter circuit that convert DC to AC.PWM techniques is used here for the Variable Ac output by using this we can easily maintain the Harmonics in easy manner and controllable feature this is another advantages for this circuit.

\section{SIMULATION RESULTS}

The multilevel H-bridge cascade system model implemented was tested both using simulation and hardware. The simulation was carried using MATLAB programming for computing. The hardware implementation was carried using various components such as the Power supply, Driver circuits and Microcontroller Unit

MATLAB is a high-performance language for technical computing. It integrates computation, visualization and programming in an easy-to-use environment where problems and solutions are expressed in familiar mathematical notation. Typical uses include

Math and computation

Algorithm development

Data acquisition

Modelling, simulation, and prototyping Data analysis.

\section{SIMULATION MODEL}

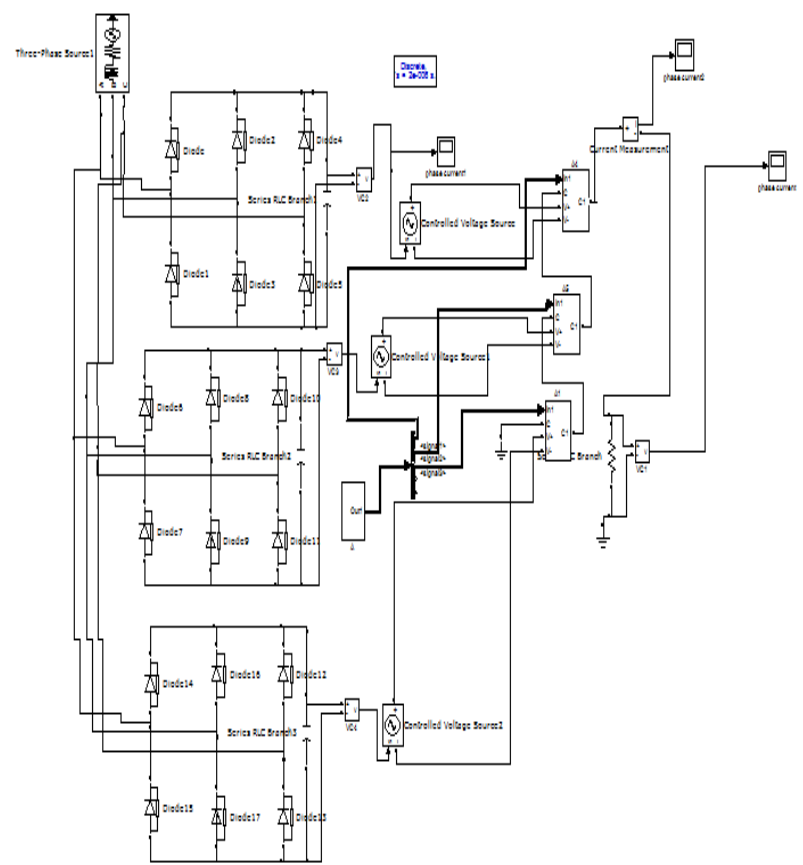

Figure 3 simulation circuit of seven level cascade h-bridge inverter

The simulation circuit for the seven level cascade $\mathrm{H}$ Bridge inverter is shown in the figure 3 . The circuit diagram consist of 3 stepdown transformer and 1 stepup transformer.In this stepdown transformer the $220 \mathrm{~V}$ AC supply voltage is stepdown to $12 \mathrm{~V}$ AC supply. The rectifier circuit that converts $\mathrm{AC}$ to $\mathrm{DC}$ for this purpose we use the half bridge rectifier circuits. This DC supply is given to the inverter circuit.
The inverter circuit consist of 3H-Bridge MOSFET inverter and 12 switches.The gate pulse for each MOSFET is given through the microcontroller in which PWM sinusoidal techniques is used.By use this we can reduces the Harmonics, clarity in the O/P waveform and reduction in the conversional losses these are the main advantages present in the PWM techniques. The output from the Inverter is stepup by transformer and given to the load.

\section{SUBSYSTEM}

The various subsystem blocks for the seven level cascade $\mathrm{H}$-Bridge inverter is shown in the figure 4.

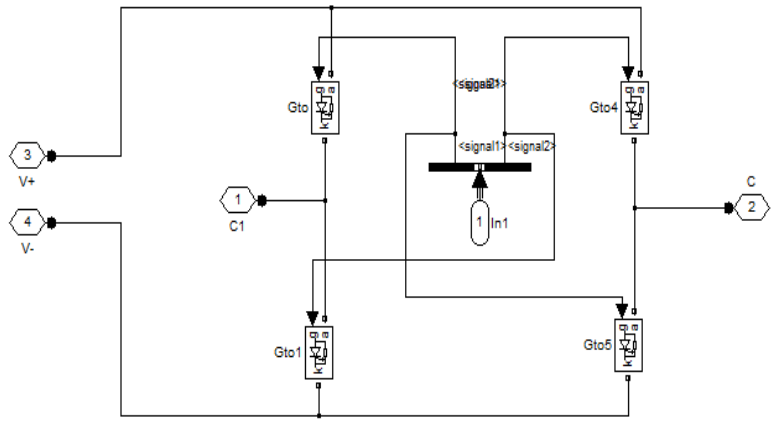

Figure 4 sub-system A1 block of 7level h-bridge inverter

\section{CASCASED MULTILEVEL INVERTER OUTPUT}

By the switching sequence the output of the seven level cascaded inverter output is obtained as shown in fig 5

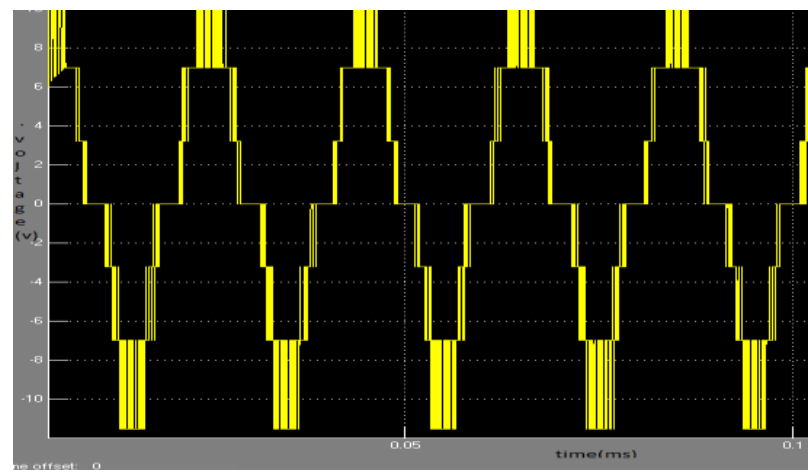

Fig 5Cascaded Inverter output voltage

In Cascaded Inverter output voltage, the voltage levels are equal and accurate. This topology provides a modularized output with the complete eradication of clamping diodes and storage capacitors which are used in the previous topologies. This makes the entire circuit very compact and thus this method is chosen for hardware implementation.

\section{Harmonic Analysis}

The FFT analysis of cascade multilevel inverter from the mat lab analysis has low THD value when compared to the other two topologies.

The third and fifth order harmonics are a major concern in any power system since it cannot be eliminated by the usage of capacitors. Thus for an efficient system these harmonics has to be as least as possible. 
INTERNATIONAL JOURNAL OF INNOVATIVE RESEARCH IN ELECTRICAL, ELECTRONICS, INSTRUMENTATION AND CONTROL ENGINEERING Vol. 3, Issue 10, October 2015

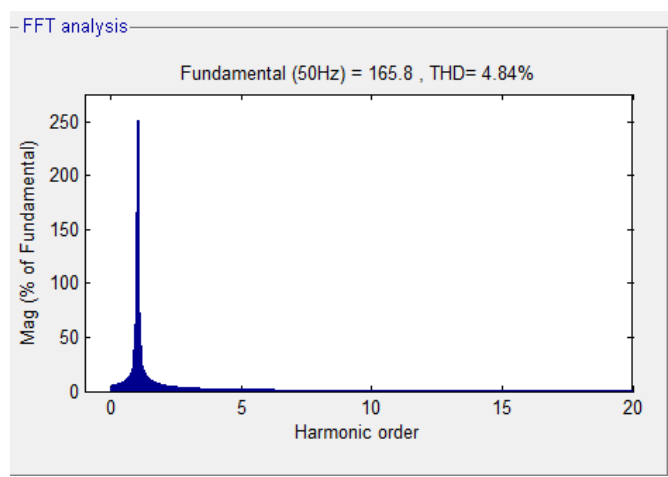

Fig 6 FFT analysis of cascaded multilevel inverter

\section{HARDWARE RESULTS}

The principle of seven level multi converters is converting the dc input supply to ac output supply. The input power supply is ac and it is converted using rectifier this will reduce losses, the harmonic also reduced.

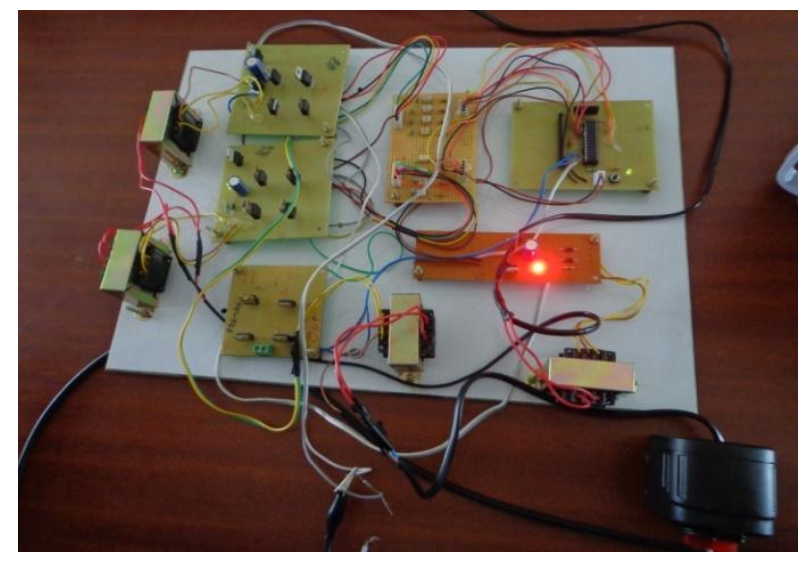

Figure 7 Hardware Design

The power supply unit will provide the power to the three step-down transformers which will give the power of 12 voltage .the hardware kit having three H-bridges the transformer output will go to this H-bridge inverter and the rectifier on it will rectify it then the power will go to capacitor for reducing the ripples then it will go to the voltage regulator then the supply will go to the inverter The controller will control the gate pulses and optocoupler will give isolated pulses to the MOSFET.

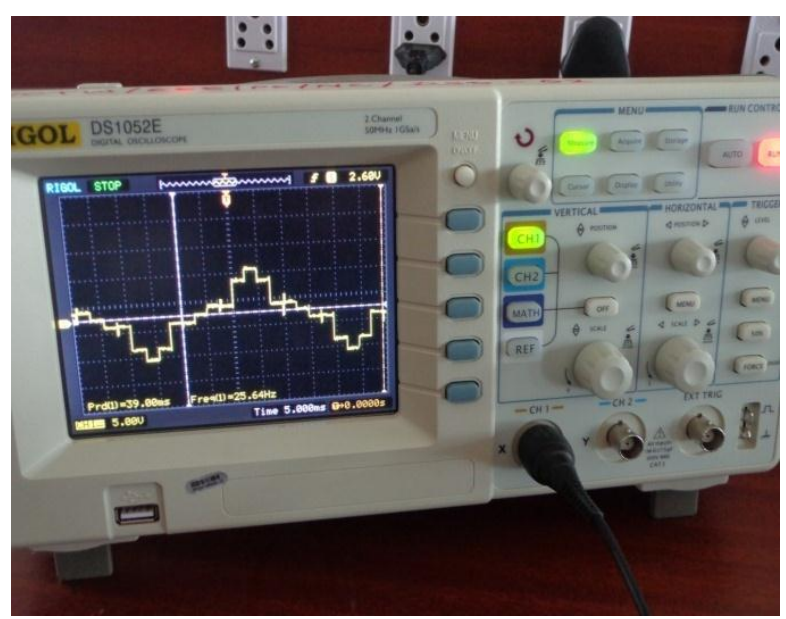

Figure 8 DSO Output

\section{CONCLUSION}

This project gives clear idea about the "Harmonic minimization in distribution network using cascade $\mathrm{H}$ bridge multilevel inverter". The proposed MLI has three $\mathrm{H}$-bridge inverter in order to achieve the output voltage of 7-level. The output waveform of the MLI was stepped wave with 7-level. Comparing to the conventional 5-level inverter the harmonics are reduced in the 7-level inverter. The output voltage level and power density was increased in the proposed system. Since, the cascaded inverter was implemented in hardware by considering both the economic and technical advantages over the other two topologies.

\section{REFERENCES}

M. Abolhassani, R. Edwards, T. Keister, A. Skorcz, E. Ledezma, and C. H. Liao, "Modular multi-pulse transformer rectifier for use in symmetric multi-level power converter," U.S. Patent pending, Pub. No. US 20100073970 A1, Mar. 25, 2010

2. M. Abolhassani, R. Edwards, T. Keister, A. Skorcz, E. Ledezma, C. H. Liao, "Modular multi-pulse transformer rectifier for use in asymmetric multi-level power converter," U.S. Patent 7830681 , Nov. 9, 2010.

3. B. S. Suh, G. Sinha, M. D. Manjrekar, and T. A. Lipo, "Multilevel power conversion-An overview of topologies and modulation strategies," in Proc. OPTIM, 1998, AD-11-AD-24

4. Z. Du, L. M. Tolbert, B. Ozpineci, and J. N. Chiasson, "Fundamental frequency switching strategies of a seven-level hybrid cascaded H-bridge multilevel inverter," IEEE Trans. Power Electron., vol. 24, no. 1, pp. 25-33, Jan. 2009.

5. B. Wu, High-Power Converters and AC Drives.. Hoboken, NJ: Wiley, 2006.

6. J. Rodríguez, J. S. Lai, F. Z. Peng, "Multilevel inverters: A survey of topologies, controls, and applications," IEEE Transactions on Industrial Electronics, vol.49, no.4, pp.724-738, 2002.

7. K. Ichikawa, "Multiple inverter system," U.S. Patent 6229722 B1, May 8, 2001.

8. P. W. Hammond, "A new approach to enhance power quality for medium voltage AC drives," IEEE Trans. Ind. Appl., vol. 33, no. 1, pp. 202- 208,Jan./Feb. 1997.

IEEE Recommended Practices and Requirements for Harmonic Control in Electrical Power Systems, IEEE Standard 519-1992, Jun. 1992

10. Agelidis, V.G. and M. Calais, 1998. Application specific harmonic performance evaluation of multicarrier PWM techniques. Proc. IEEE PESC'98, 1: 172-178. 